\title{
A multi-path switched-capacitor-inductor hybrid DC-DC converter with reduced inductor loss and extended voltage conversion range
}

\author{
Qiaobo Ma ${ }^{1}$, Xiongjie Zhang', Yang Jiang' ${ }^{1 a)}$, Katsuhiro Hata ${ }^{2}$, Makoto Takamiya ${ }^{2}$, Man-Kay Law ${ }^{1}$, Pui-In Mak ${ }^{1}$, and \\ Rui P. Martins ${ }^{1,3}$
}

\begin{abstract}
This work proposes a hybrid DC-DC converter using a multi-path switched-capacitor-inductor (MPSCI) topology. Assisted by switchedcapacitor branches, it features voltage-conversion range extension and inductor power loss reduction, and hence improving the conversion efficiency. Implemented in a $180-\mathrm{nm}$ CMOS process, the proposed converter can regulate an output voltage of $1.8 \sim 3.3 \mathrm{~V}$ from a 5 -V input bus voltage using a $4.7 \mu \mathrm{H}$ inductor $(\mathrm{DCR}=240 \mathrm{~m} \Omega)$ at a switching frequency of $800 \mathrm{kHz}$. It achieves a peak conversion efficiency of $93.7 \%$ when delivering an output current of $1 \mathrm{~A}$. Under a maximum loading of $3 \mathrm{~A}$, this design still attains an efficiency of up to $85.8 \%$. Furthermore, in contrast to the existing dual-path and conventional buck topologies, the proposed converter realizes an inductor loss reduction up to 3.3 and 5.4 times, respectively, at the maximum loading conditions.

key words: DC-DC converter, DCR loss, hybrid topology, multi-path, switched-capacitor, voltage conversion range

Classification: Integrated circuits (analog)
\end{abstract}

\section{Introduction}

In recent years, the fast-growing demand for portable intelligent devices powered by low-voltage buses or batteries requires high-efficiency power converters with a small form factor [ $\left[\begin{array}{lllllll}1 & 2 & 3 & 4 & 5 & 6 & 7\end{array}\right]$. The improvement of the functionality and performance of the loading systems brings more challenges to the DC-DC converter design to suffice the rising loading current requirements. Existing inductorat-output topologies, such as buck or 3-level topologies [1 2 89101112131415161718 ], suffer from significantly climbed-up conduction loss on the power inductor when delivering a high loading current with a compact system volume. It is mainly due to the loss of inductor parasitic DC resistance (DCR) and can be critical for achieving high power conversion efficiency (PCE). As the tradeoff, a bulky

\footnotetext{
${ }^{1}$ State Key Laboratory of Analog and Mixed-Signal VLSI, Institute of Microelectronics, and the Faculty of Science and Technology - ECE, University of Macau, Macao 999078, P. R. China.

${ }^{2}$ Institute of Industrial Science, The University of Tokyo, Tokyo 153-8505, Japan.

${ }^{3}$ Instituto Superior Técnico, Universidade de Lisboa, 1049001 Lisbon, Portugal.

a) timjiang@um.edu.mo
}

DOI: $10.1587 /$ elex.18.20210405

Received September 26, 2021

Accepted October 08, 2021

Publicized October 18, 2021

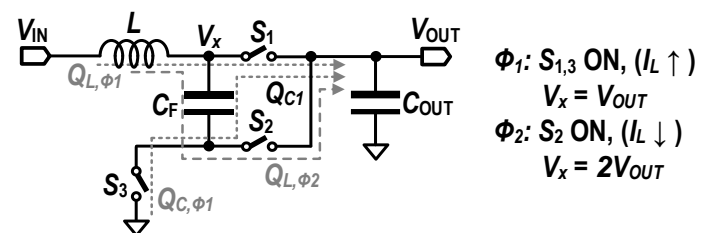

(a)

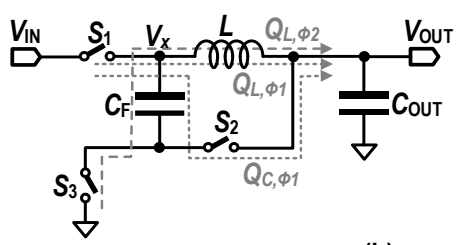
$\Phi_{1}: S_{1,2}$ ON, $\left(I_{L} \uparrow\right)$ $V_{x}=V_{I N}$
$\Phi_{2}: S_{3} \mathrm{ON},\left(I_{L} \downarrow\right)$
$V_{x}=V_{I N}-V_{O U T}$

(b)

Fig. 1 Existing DPSCI topologies with VCR $=1 /(2-\mathrm{D}),(0<\mathrm{D}<1)$ reported in (a) [1] and (b) [20].

inductor with a smaller DCR can contribute to the efficiency improvement by, however, scarifying the system compactness [2 3441316 19]. Within a specific volume, the DCR increases with the inductance and can be as high as hundreds of milliohms, which is dramatically higher than the switch conducting resistance $\left(R_{\mathrm{ON}}\right)$ and hence introduces considerable influence to the overall PCE. A common issue with the inductor-at-output converters is that the total output current ( $I_{\text {OUT }}$ ) flows through the inductor, leading to a high $\mathrm{I}^{2} \mathrm{R}$ loss (thermal dissipation) when delivering a large $I_{\text {OUT }}$.

To limit the converter size and simultaneously reduce the power dissipation on the inductor, a practical solution is to reduce the inductor current by adding more currentconducting paths to the output, as demonstrated in [ [ $\begin{array}{llll}1 & 20 & 21\end{array}$ $22 \quad 2324$ 25]. For example, Fig. 1 shows two existing dualpath (DP) switched-capacitor-inductor hybrid topologies proposed in $\left[\begin{array}{ll}1 & 20\end{array}\right]$. These topologies reduce the inductor current by delivering a part of IOUT through the switchedcapacitor (SC) branches. However, these DP topologies suffer from a restricted voltage conversion ratio (VCR) of $1 / 2$ to 1 due to the constraints on inductor charging and discharging voltages, limiting their application of converting a $5-\mathrm{V}$ bus voltage to a $1.8-\mathrm{V}$ load supply. Although this issue can be resolved by adding an extra power switch and one more switching phase, as discussed in [20], the effectiveness of the inductor current reduction degrades with enlarging the step-down VCR, resulting in a 
IEICE Electronics Express, Vol.xx, No.xx, xx-xx

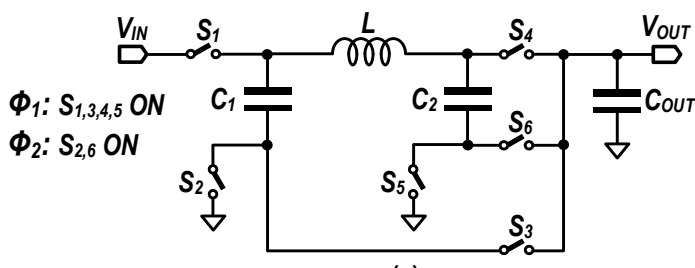

(a)
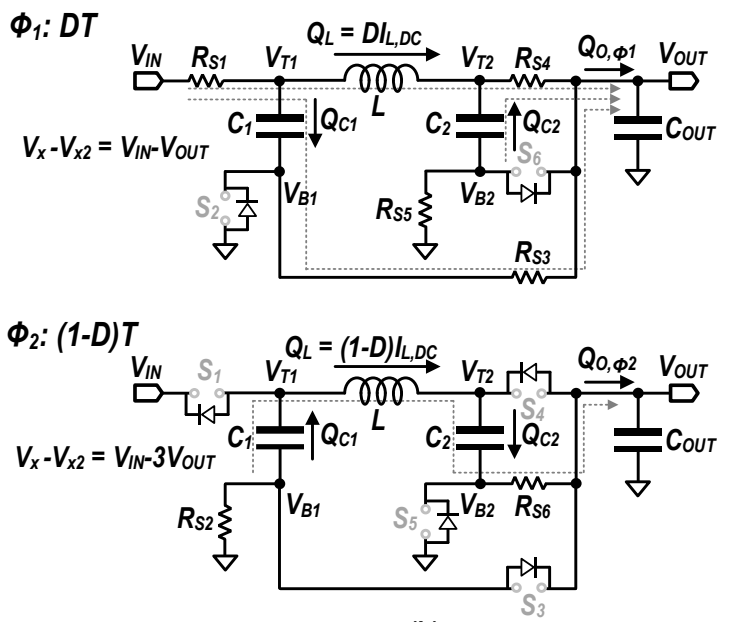

(b)

Fig. 2 (a) The Proposed MPSCI converter topology, and (b) its two-phase operating principle.

noticeable PCE drop.

This work proposes a multi-path switched-capacitorinductor (MPSCI) converter topology, addressing the inductor DCR loss and VCR range limitation in high loading current conversions. It consists of a single inductor and dual flying capacitors to realize an extended step-down ratio of $1 / 3$ to 1 , featuring enhanced inductor current reduction in a higher-step-down (closer to 1/3) VCR condition.

\section{The proposed MPSCI converter topology}

\subsection{Steady-State Operations}

Regarding the inductor-voltage analysis for the existing DP topologies depicted in Fig. 1, the VCR limitation is determined by the voltage across the inductor in both charging and discharging phases. Based on the inductor current balance in steady state, applying $V_{\mathrm{IN}}-2 V_{\text {OUT }}<0$ in $\Phi_{2}$ for the inductor discharging indicates that $V_{\mathrm{OUT}} / V_{\mathrm{IN}}>1 / 2$ for both topologies shown in Fig. 1. Accordingly, a higher voltage difference across the inductor during its current decaying phase can give a lower VCR boundary and, hence, realize a wider conversion range. An effective way to extend the step-down conversion range is to increase the voltage difference across the inductor from $\left(V_{\mathrm{IN}}-2 V_{\mathrm{OUT}}\right)$ to $\left(V_{\mathrm{IN}}-\right.$ $3 V_{\text {OUT }}$ ) during $\Phi_{2}$ by using two SC cells, coming up with the proposed MPSCI topology as illustrated in Fig. 2. According to the operating states shown in Fig. 2(b), the steady-state capacitor voltages on $C_{1}$ and $C_{2}$ are $\left(V_{\mathrm{IN}}-V_{\mathrm{OUT}}\right)$ and $V_{\mathrm{OUT}}$, respectively. Based on the steady-state volt-second-balance relation of the inductor, the corresponding VCR expression

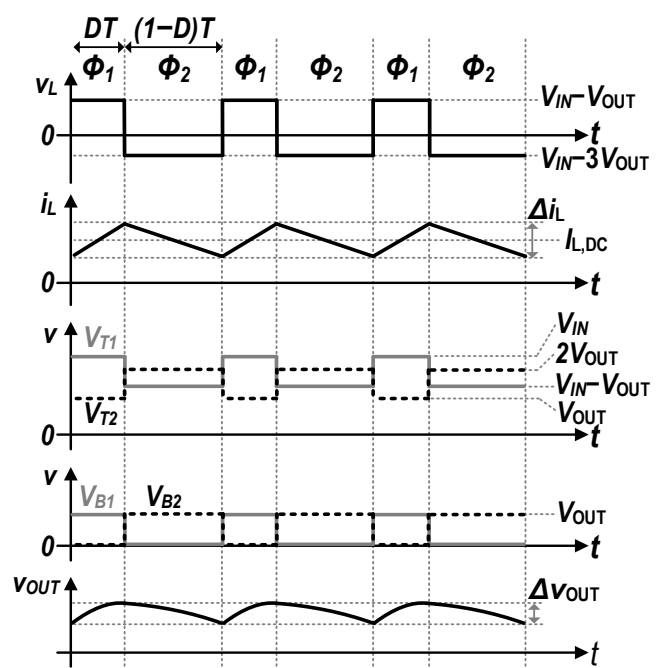

Fig. 3 Conceptual waveforms for the steady-state operation of the proposed MPSCI topology.

is:

$$
\begin{gathered}
M=\frac{V_{\text {OUT }}}{V_{I N}}=\frac{1}{3-2 D}, 0<D<1 . \\
D=\frac{3 M-1}{2 M}
\end{gathered}
$$

As observed, the duty ratio $D$ varying between 0 and 1 results in a VCR from $1 / 3$ to 1 .

During $\Phi_{1}$, the current $i_{\mathrm{L}}$ ramps up as the inductor voltage $V_{\mathrm{L}, \Phi 1}=V_{\mathrm{IN}}-V_{\text {OUT }}$. Paralleled with the inductor, $C_{1}$ is charged up to the same voltage as the $V_{\mathrm{L}, \Phi 1}$. At the same time, capacitor $C_{2}$ is discharged by the converter output and, hence, $V_{\mathrm{C} 2}=V_{\text {OUT }}$ in steady-state. During $\Phi_{2}, L, C_{1}$, and $C_{2}$ are connected in series. Thus, by applying a small ripple approximation to the inductor, the total charges flowing to $C_{1}$ and $C_{2}$ are

$$
Q_{C 1}=Q_{C 2}=(1-D) T I_{L, D C},
$$

where $I_{\mathrm{L}, \mathrm{DC}}$ is the DC average of the $i_{\mathrm{L}}$, and $T$ is the switching period. $i_{\mathrm{L}}$ decays as $V_{\mathrm{L}, \Phi 2}=V_{\mathrm{IN}}-3 V_{\mathrm{OUT}}$ during $\Phi_{2}$, while the charging/discharging states for $C_{1}$ and $C_{2}$ are opposite to $\Phi_{1}$. According to the above operations, three current-conducting branches (through $L, C_{1}$, and $C_{2}$ ) are connected to the output during $\Phi_{1}$, realizing a multi-path output-charge delivery. In contrast to single-path structures, the proposed scheme effectively reduces the $I_{\mathrm{L}, \mathrm{DC}}$. Based on charge conservation and the expression in (2), the $I_{\mathrm{L}, \mathrm{DC}}$ is derived as below:

$$
\begin{aligned}
& T I_{L, D C}+Q_{C 1}+Q_{C 2}=Q_{O U T} \\
& \quad \Rightarrow I_{L, D C}=\frac{I_{L O A D}}{3-2 D}=M I_{L O A D} .
\end{aligned}
$$

From above, the inductor current can be reduced by a factor of $M$ when compared with the existing inductor-at-output topologies.

Fig. 3 exhibits the conceptual waveforms for the steady- 
state operations of the proposed topology. According to the information of $V_{\mathrm{T} 1}-V_{\mathrm{T} 2}$ in either $\Phi_{1}$ or $\Phi_{2}$, the inductor current ripple $\Delta i_{\mathrm{L}}$ has the following form:

$$
\Delta i_{L}=\frac{\left(V_{I N}-V_{O U T}\right) D T}{L}=\frac{T V_{O U T}}{L} \frac{(3 M-1)(1-M)}{2 M^{2}} .
$$

From (4), the peak value of the $\Delta i_{\mathrm{L}}$ occurs at $M=0.5$, and the corresponding maximum inductor current ripple amplitude equals $0.5 T V_{\text {OUT }} / L$. Thus, in the conversion range from $1 / 3$ to $1 / 2$, the proposed topology achieves a lower inductor current for $\mathrm{DC}$ and $\mathrm{AC}$ components than a buck.

\subsection{Conduction Loss Analysis}

Under heavy load conditions, the conduction losses from inductor DCR and switches $R_{\mathrm{ON}}$ dominate the converter efficiency [26 27]. The corresponding theoretical loss analysis is as below

$$
I_{L, r m s}=\sqrt{\frac{1}{T} \int_{0}^{T} i_{L}^{2}(t) d t}=\sqrt{I_{L, D C}{ }^{2}+\frac{\Delta I_{L}^{2}}{12}} .
$$

By substituting (3) into (5), we can derive the loss power expression accordingly, i.e.,

$$
\begin{aligned}
P_{\text {loss }, L} & =I_{L, r m s}^{2} R_{L, D C} \\
& =R_{L, D C}\left\{M^{2} I_{\text {OUT }}^{2}+\frac{1}{12}\left[\frac{V_{\text {OUT }}}{L f_{S}} \frac{(3 M-1)(1-M)}{2 M^{2}}\right]^{2}\right\} .
\end{aligned}
$$

From (6), the DC part of the conduction loss with the MPSCI is $M^{2}$ times smaller than that of the inductor-at-output converters. Besides, the AC part of the conduction loss due to the inductor current ripple decreases with $M$. From Fig. 3, the charge amount $Q_{\mathrm{C} 1,2}$ flowing through the capacitors $C_{1,2}$ equals to the $Q_{\mathrm{L}}$ during $\Phi_{2}$, estimated as

$$
Q_{C 1,2}=(1-D) T I_{L, D C}
$$

According to the steady-state charge conservation with the capacitor, the average current through the $C_{1,2}$ during $\Phi_{1}$ is

$$
I_{C 1,2, \phi 1}=\frac{Q_{C 1,2}}{D T}=\frac{(1-D)}{D} I_{L, D C}
$$

Based on (8) and the operating states shown in Fig. 2(b), we can estimate the passing current through different switches in each phase, as:

$$
\begin{gathered}
\Phi_{1}:\left\{\begin{array}{c}
I_{R S 1}=\frac{1}{D} I_{L, D C} \\
I_{R S 3}=\frac{(1-D)}{D} I_{L, D C} \\
I_{R S 4}=\frac{1}{D} I_{L, D C} \\
I_{R S 5}=\frac{(1-D)}{D} I_{L, D C}
\end{array}\right. \\
\Phi_{2}:\left\{\begin{array}{l}
I_{R S 2}=I_{L, D C} \\
I_{R S 6}=I_{L, D C}
\end{array}\right.
\end{gathered}
$$

where $I_{\mathrm{RSi}}$ represents the passing current through the switch $S_{i}$. The overall switch conduction loss expression is:
Table I Topology modeling parameters

\begin{tabular}{c|c|c|c|c|c|c|c}
\hline$V_{I N}$ & $f_{S W}$ & $I_{\text {OUT }}$ & $\mathrm{L}$ & $C_{F}$ & $C_{\text {OUT }}$ & $R_{\text {ON }}$ & $\mathrm{DCR}$ \\
\hline $5 \mathrm{~V}$ & $500 \mathrm{kHz}$ & $2 \mathrm{~A}$ & $4.7 \mu \mathrm{H}$ & $10 \mu \mathrm{F}$ & $20 \mu \mathrm{F}$ & $10 \mathrm{~m} \Omega$ & $250 \mathrm{~m} \Omega$ \\
\hline
\end{tabular}

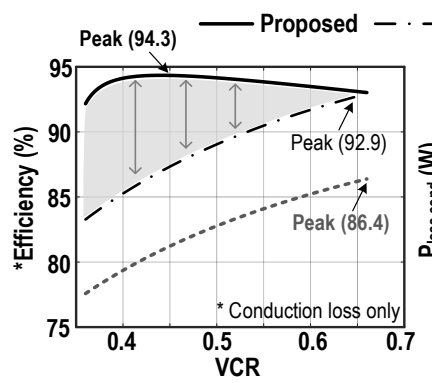

(a)

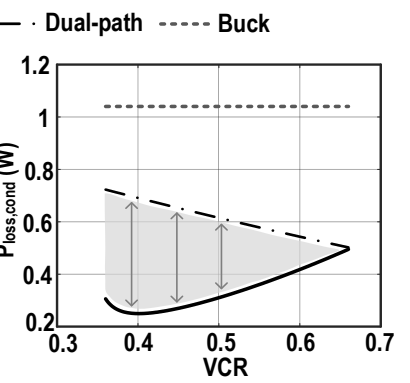

(b)
Fig. 4 Modeling results and comparison between the proposed and the existing topologies on (a) conduction-loss-only conversion efficiency and (b) the conduction loss power using the parameters given in Table I.

$$
\begin{gathered}
P_{\text {loss }, S W}=D\left(I_{R S 1}^{2} R_{S 1}+I_{R S 3}^{2} R_{S 3}+I_{R S 4}^{2} R_{S 4}+I_{R S 5}^{2} R_{S 5}\right) \\
+(1-D)\left(I_{R S 2}^{2} R_{S 2}+I_{R S 6}^{2} R_{S 6}\right) \\
=I_{L, D C}{ }^{2}\left[\frac{1}{D}\left(R_{S 1}+R_{S 4}\right)+\frac{(1-D)^{2}}{D}\left(R_{S 3}+R_{S 5}\right)+(1-\right. \\
\left.D)\left(R_{S 2}+R_{S 6}\right)\right]
\end{gathered}
$$

where $R_{\mathrm{Si}}$ denotes the switch-on resistance of the switch $S_{i}$, as shown in Fig. 2. Based on the above theory, we can establish an analytical model of the conduction loss power to evaluate and compare the performance of different topologies. Using the corresponding parameters given in Table I, we can obtain the modeling results, as displayed in Fig. 4, for the proposed MPSCI, existing DP, and the conventional buck topologies. From the highlighted region in the figures, the efficiency and power loss difference between the proposed and the existing topologies become more noticeable as the VCR enlarging.

\section{Converter design and implementation}

The overview implementation of the proposed MPSCI converter system composes of the power stage, gate drivers, and the feedback control loop, as shown in Fig. 5. All the power switches are based on $5-\mathrm{V}$ transistors in a $180-\mathrm{nm}$ CMOS process to ensure operating reliability. Regarding the power passive selection, the capacitance of $C_{1}$ and $C_{2}$ is $10 \mu \mathrm{F}$, and $C_{\text {OUT }}$ is $20 \mu \mathrm{F}$ with an equivalent series resistance (ESR) of $10 \mathrm{~m} \Omega$ for each. The converter adopts an inductor of $4.7 \mu \mathrm{H}$ with $\mathrm{DCR}=240 \mathrm{~m} \Omega$ and a dimension of $2.5 \mathrm{~mm} \times$ $2 \mathrm{~mm} \times 1 \mathrm{~mm}$. It features regulating a $V_{\text {OUT }}$ of $1.8 \sim 3.3 \mathrm{~V}$ from an input of $5 \mathrm{~V}$ based on voltage-mode PWM control. A typical type-III compensated error amplifier (EA) is adequate for stabilizing the load regulation control loop. For proper switch on/off operations, the gate driving voltage for all the switches is $5 \mathrm{~V}$. Except for the input switch $\mathrm{S}_{1}$, which is implemented by a PMOS transistor to ease the driving 


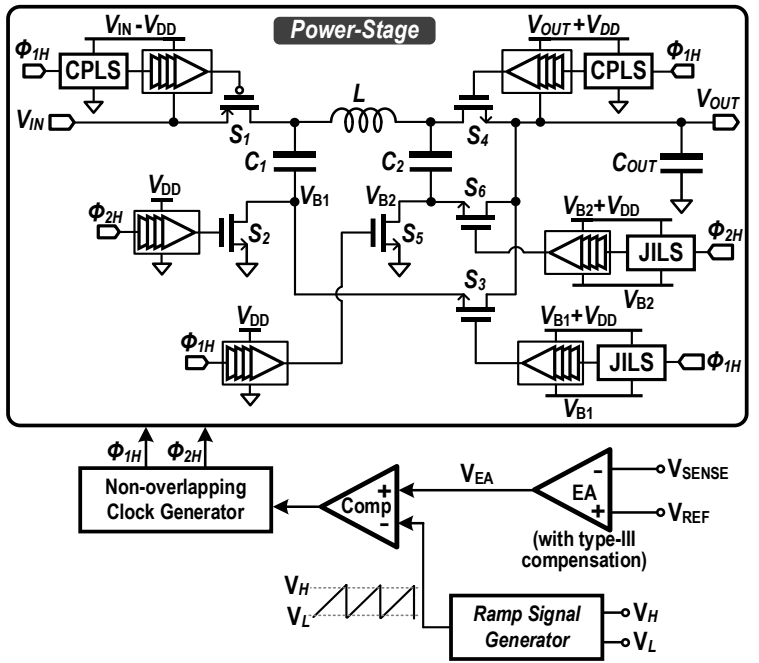

Fig. 5 Implementation overview of the proposed converter system.
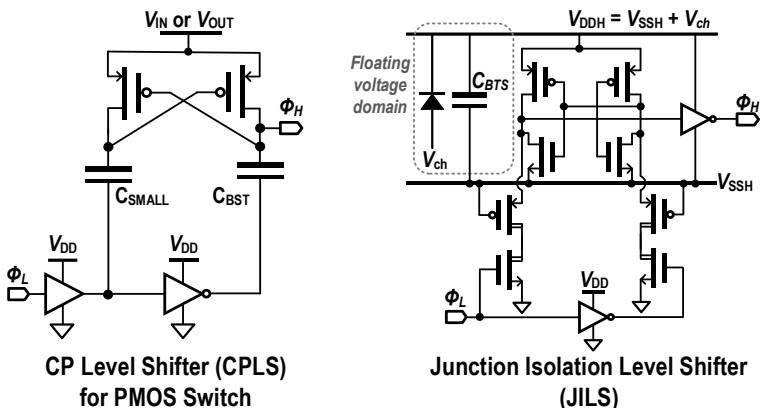

(JILS)

Fig. 6 Circuit implementation of the level shifters adopted in the proposed converter for switch control.

requirement, all the other switches are implemented by NMOS for better conductance and lower silicon area.

We designed the gate driver circuits in three categories for different power switches according to the individual operating voltage conditions. Specifically, $\mathrm{S}_{2}$ and $\mathrm{S}_{5}$ are GND-connected switches, an inverter-based buffer chain with a stage size increase by a factor of 10 that ensures an effective fan-out is applicable for the gate driving. A starving-resistor-based buffer is used to drive the last stage of the buffer chain to prevent shoot-through current. Due to the fixed-DC-terminal property with the operation of $\mathrm{S}_{1}$ and $\mathrm{S}_{4}$, charge pump-based level shifters (CPLS), as given in Fig. 6 , with different types of cross-coupled transistors can be used to fulfill the driving requirements [ [28 29 30]. Considering $\mathrm{S}_{1}$ as an example, the generated turn-on and off gate-driving voltage are $V_{\mathrm{IN}}-V_{\mathrm{DD}}$ and $V_{\mathrm{IN}}$, respectively. A similar control principle applies to $S_{4}$. Since $S_{3}$ and $S_{6}$ are floating switches (i.e., with no terminal connected to a DC voltage), bootstrapping driving techniques are necessary to generate floating voltage domains for the individual switches. The junction isolation level shifter (JILS), as shown in Fig. 6, shifts up the clock signal from $0 \sim V_{\mathrm{DD}}$ to $V_{\mathrm{SSH}} \sim\left(V_{\mathrm{SSH}}+V_{\mathrm{ch}}\right)$ domain, $V_{\mathrm{ch}}=V_{\mathrm{DD}}$ according to the node switching voltages of $V_{\mathrm{B} 1}$ and $V_{\mathrm{B} 2}$.

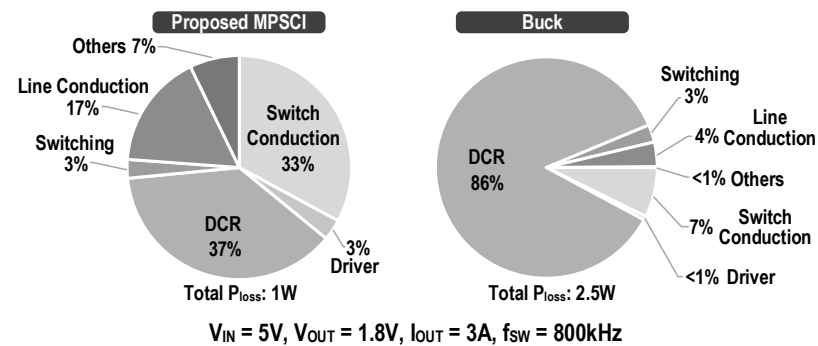

Fig. 7 Complete loss breakdown comparison between the proposed MPSCI and conventional buck converters under a specific operating condition of $\mathrm{V}_{\mathrm{OUT}}=1.8 \mathrm{~V}$ and $\mathrm{I}_{\mathrm{OUT}}=3 \mathrm{~A}$.

\section{Simulation results}

The converter design validation is through full-transistorlevel simulation at a switching frequency of $f_{\mathrm{Sw}}=800 \mathrm{kHz}$. The concerned losses include inductor DCR loss, switch $R_{\mathrm{ON}}$ conduction loss, switching loss, switch gate driving loss, and shunt loss from the control building blocks. Considering the line-conduction loss caused by the practical parasitic effect, each power switch is in series with a line impedance of $\sim 10 \mathrm{~m} \Omega$. A simulated loss-power breakdown is summarized in Fig. 7 for a $5 \mathrm{~V}$-to-1.8V conversion with an output current of $3 \mathrm{~A}$. It validates that the proposed MPSCI converter achieves improved overall efficiency compared with a buck converter implemented with the same total switch area and power inductor. From the pie chart, in contrast to the buck, the DCR loss reduction in the proposed converter is significant, and hence it attains a higher efficiency under a heavy load condition.

Fig. 8 shows the simulated efficiency of the proposed MPSCI converter under different loading conditions and VCRs. From Fig. 8(a), this design ensures a PCE above 90\% over a conversion range of $5 \mathrm{~V}$ to $1.8-3.3 \mathrm{~V}$ under $1 \mathrm{~A}$ loading current. The peak efficiency can be up to $93.7 \%$. When delivering a current of $2 \mathrm{~A}$, the total conduction loss increases, and the converter can still reach a $90 \%$ peak efficiency and maintain the overall PCE above $86 \%$. Targeting on a maximum loading of $3 \mathrm{~A}$, the simulated result demonstrates a peak efficiency of $85.8 \%$. Fig. 8(b) displays the simulated curves of PCE and DCR loss power together with a comparison between the proposed converter and the existing ones under a continuous loading current range and different $V_{\text {OUT }}$ of 1.8 and $2.5 \mathrm{~V}$. In contrast to the existing DP and conventional buck converters, the proposed MPSCI realized a DCR loss reduction up to 3.3 and 5.4 times and hence a PCE benefit of $7.1 \%$ and $15 \%$, respectively, at a 1.8 $\mathrm{V}$ output. As the increase of the $V_{\text {OUT }}$ level, the DCR loss power takes less proportion among the total loss. On the other hand, although the relative DCR loss advantage attained by the proposed topology shrinks as the increase of the $\mathrm{D}$, according to (3), it still realize a PCE advantage up to $2.3 \%$ and $11 \%$, and a DCR loss reduction up to 1.5 and 3 


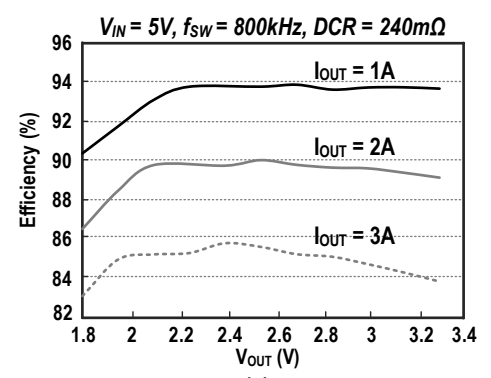

(a)
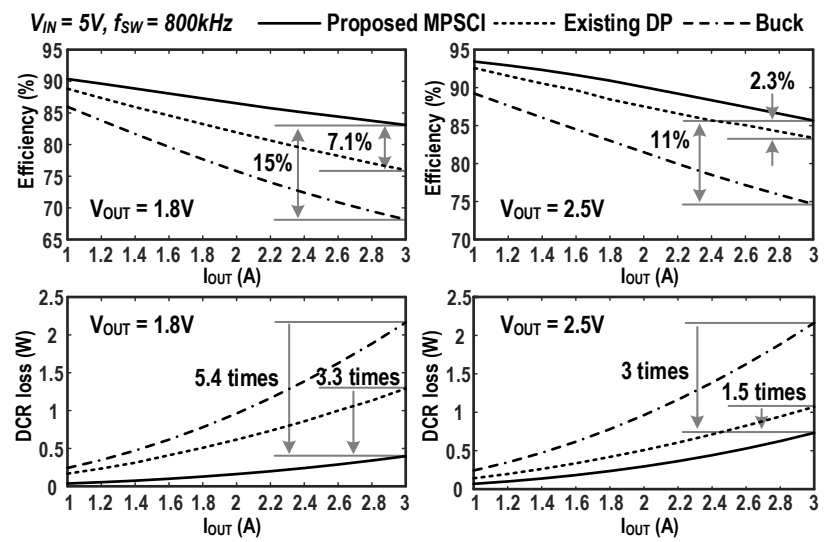

(b)

Fig. 8 Simulated results and comparison between the proposed MPSCI and the existing topologies on (a) the efficiency versus $V_{\text {OUT }}$ under different $I_{\text {OUT }}$ and (b) the efficiency and DCR loss versus $I_{\text {OUT }}$ under different $V_{\text {OUT }}$.

times when compared with the existing DP and conventional buck converter, respectively.

Fig. 9 shows the frequency-domain characteristics of the proposed converter. From the Bode plot, the power stage double poles locate around $16 \mathrm{kHz}$, and the loop phase margin is $54^{\circ}$ after the compensation. In contrast, the power stage phase margin is only $7^{\circ}$ in the open-loop condition. Accordingly, the type-III compensator is adequate for stabilizing the voltage-mode control loop of the implemented converter system.

Fig. 10 depicts the time-domain transient waveforms of the proposed converter under a step response with the loading current from 0.5 to $3 \mathrm{~A}$. A $\sim 10 \% V_{\text {OUT }}$ overshoot/undershoot happens during the step-current transition and the recovery time is about $35 \mu$ s. As observed, during the dead-time, the body diode of the power MOSFETs in the inductor current path is in forward conducting to provide a freewheeling path for the $\mathrm{I}_{\mathrm{L}}$, causing the diode voltage drop observed in the waveform. Besides, the $V_{\text {OUT }}$ ripple is about $30 \mathrm{mV}$, which is majorly due to the ESR influence mainly.

The overall performances of the proposed converter are summarized in Table II and compared to the state of the art. From the table, this work achieves high efficiency and a high loading current level using a large-DCR inductor. In addition, the proposed topology achieves a higher inductor current reduction theoretically under a high step-down condition and hence demonstrates an advantage of delivering heavier
IEICE Electronics Express, Vol.xx, No.xx, xx-xx

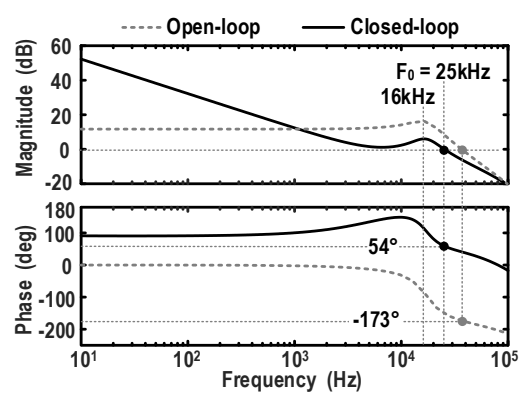

Fig. 9 Frequency-domain characteristics of the proposed converter in open and closed-loop conditions.

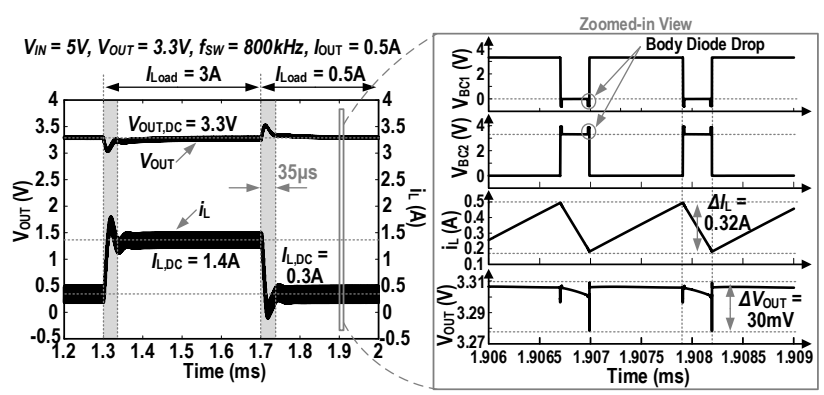

Fig. 10 Simulated time-domain waveforms of proposed converter with step responses of the output current as well as the steady-state power stage internal waveforms.

\begin{tabular}{|c|c|c|c|c|c|}
\hline & ISSCC'15 [15] & APEC'17 [2] & ISSCC'17 [14] & JSSC'19 [20] & This Work \\
\hline Process & $0.35 \mathrm{um}$ & Discrete & $28 \mathrm{~nm}$ & $0.18 \mathrm{um}$ & 0.18um \\
\hline Topology & Buck & S-hybrid & 3-Level & DPDC & MPSCI \\
\hline $\mathrm{V}_{\mathrm{IN}}[\mathrm{V}]$ & $2.7-4.5$ & 5 & $3-4.5$ & 4.5 & 5 \\
\hline $\mathrm{V}_{\text {OUT }}[\mathrm{V}]$ & 2 & $3.3-3.8$ & $0.8-1.45$ & $0.8-4$ & $1.8-3.3$ \\
\hline $\begin{array}{l}\text { Max. output } \\
\text { current [A] }\end{array}$ & 1 & 3.9 & 1 & 1.6 & 3 \\
\hline $\begin{array}{l}\mathrm{f}_{\mathrm{SW}} \text { frequency } \\
{[\mathrm{MHz}]}\end{array}$ & 1 & 2 & 3 & 1 & 0.8 \\
\hline $\mathrm{I}_{\mathrm{L}, \mathrm{DC}}$ reduction & No & $\begin{array}{c}\text { Yes } \\
\mathrm{I}_{\mathrm{L}}=\mathrm{I}_{\mathrm{OUT}} /(2-\mathrm{D})\end{array}$ & No & $\begin{array}{c}\text { Yes } \\
\mathrm{I}_{\mathrm{L}}=\mathrm{I}_{\text {OUT }} /(2-\mathrm{D})\end{array}$ & $\begin{array}{c}\text { Yes } \\
I_{L}=I_{\text {OUT }} /(3-2 D)\end{array}$ \\
\hline $\begin{array}{l}\text { \# of discrete } \\
\text { components }\end{array}$ & $\begin{array}{c}1 \mathrm{~L}, 1 \mathrm{C} \\
(4.7 \mu \mathrm{H}, 10 \mu \mathrm{F})\end{array}$ & $\begin{array}{c}\text { USB } 3.0 \\
\text { Cable, } 4 \mathrm{C}(10 \\
\mu \mathrm{F} \times 4)\end{array}$ & $\begin{array}{c}1 \mathrm{~L}, 1 \mathrm{C} \\
(2.2 \mu \mathrm{H}, 2 \mu \mathrm{F})\end{array}$ & $\begin{array}{c}1 \mathrm{~L}, 2 \mathrm{C} \\
(4.7 \mu \mathrm{H}, 10 \mu \mathrm{F} \\
\times 2)\end{array}$ & $\begin{array}{c}1 \mathrm{~L}, 3 \mathrm{C}(4.7 \mu \mathrm{H}, \\
10 \mu \mathrm{F} \times 2,20 \mathrm{uF})\end{array}$ \\
\hline Inductor DCR & $38 \mathrm{~m} \Omega$ & $141.4 \mathrm{~m} \Omega$ & NA & $250 \mathrm{~m} \Omega$ & $240 \mathrm{~m} \Omega$ \\
\hline $\begin{array}{l}\text { Efficiency @ } \\
\text { 1A loading }\end{array}$ & $88.5 \%$ & $88.0 \%$ & $80.0 \%$ & $94.0 \%$ & $93.7 \%$ \\
\hline $\begin{array}{l}\text { Full load } \\
\text { efficiency }\end{array}$ & - & 84\%@3.9A & - & 92\%@1.6A & $85.8 \% @ 3 \mathrm{~A}$ \\
\hline
\end{tabular}

loading current. It features significance for the efficiency optimization, owing to the improvement of the DCR loss.

\section{Conclusion}

This letter proposes a hybrid DC-DC converter technique that features multi-path output-charge conduction to achieve effective inductor current reduction and, therefore, a lower DCR loss. This property ensures the possibility of adopting a large-DCR power inductor with a shrunk volume in highoutput-current delivery scenarios. A systematic analysis, design, and implementation methodology have been 
discussed. Through the simulation validation, in contrast to the existing techniques, the proposed topology demonstrates an extended VCR range down to $1 / 3$ and a significant efficiency advantage when delivering a loading current up to $3 \mathrm{~A}$. Furthermore, the converter implementation attains a peak efficiency of $93.7 \%$ at $1 \mathrm{~A}$ and $85.8 \%$ at $3 \mathrm{~A}$ conditions.

\section{Acknowledgments}

This work was supported in part by the Macao FDCT Grant SKL-AMSV(UM)-2020-2022, 0148/2020/A3, and 0034/2021/A1, and in part by the Research Committee of the University of Macau Grant MYRG2020-00177-AMSV.

\section{References}

[1] G. Gabian, et al.: "Hybrid buck converter optimization and comparison for smart phone integrated battery chargers," 2018 IEEE Applied Power Electronics Conference and Exposition (2018) 2148 (DOI: 10.1109/APEC.2018.8341314).

[2] G. Seo, et al.: "An inductor-less hybrid step-down DC-DC converter architecture for future smart power cable," IEEE Applied Power Electronics Conference and Exposition (2017) 247 (DOI: 10.1109/APEC.2017.7930701).

[3] S. M. Ahsanuzzaman, et al.: "An Integrated High-Density Power Management Solution for Portable Applications Based on a Multioutput Switched-Capacitor Circuit," in IEEE Transactions on Power Electronics $31 \quad$ (2016) $4305 \quad$ (DOI: 10.1109/TPEL.2015.2474738).

[4] S. Dam, et al.: "A Hybrid, Fully-Integrated, Dual-Output DC-DC Converter for Portable Electronics," in IEEE Transactions on Power Electronics 36 (2021) 4360 (DOI: 10.1109/TPEL.2020.3019273).

[5] M. Huang, et al.: "Single-Inductor Multi-Output (SIMO) DC-DC Converters with High Light-Load Efficiency and Minimized CrossRegulation for Portable Devices," in IEEE Journal of Solid-State Circuits 44 (2009) 1099 (DOI:10.1109/JSSC.2009.2014726).

[6] Ming-Hsin Huang, et al.: "Single-inductor dual-output DC-DC converters with high light-load efficiency and minimized crossregulation for portable devices," IEEE Symposium on VLSI Circuits (2008) 132 (DOI:10.1109/VLSIC.2008.4585980).

[7] M. Huang, et al.: "Sub-1 V Input Single-Inductor Dual-Output (SIDO) DC-DC Converter with Adaptive Load-Tracking Control (ALTC) for Single-Cell-Powered Systems," in IEEE Transactions on $\begin{array}{lllll}\text { Power Electronics } & \mathbf{2 5} & \text { (2010) } & 1713 & \text { (DOI: }\end{array}$ 10.1109/TPEL.2010.2042073).

[8] C. Qing, et al.: "A tri-mode high light-load efficiency BUCK converter for mobile phone application," IEICE Electron. Express 13 (2016) 20160360 (DOI: 10.1587/elex.13.20160360).

[9] X. Liu, et al.: "Analysis and Design Considerations of Integrated 3Level Buck Converters," in IEEE Transactions on Circuits and Systems I: Regular Papers 63 (2016) 671 (DOI: 10.1109/TCSI.2016.2556098).

[10] X. Ruan, et al.: "Fundamental Considerations of Three-Level DCDC Converters: Topologies, Analyses, and Control," in IEEE Transactions on Circuits and Systems I 55 (2008) 3733 (DOI:10.1109/TCSI.2008.927218).

[11] K. Wei, et al.: "Comparative topology and power loss study for high power density and high conversion ratio integrated switching power converters," IEEE 8th Latin American Symposium on Circuits \& Systems (2017) 1 (DOI: 10.1109/LASCAS.2017.7948055).

[12] J. Moon, et al:: "Design of low-power, fast-transient-response, capacitor-less low-dropout regulator for mobile applications," IEICE Electron. Express 13 (2016) 1 (DOI: 10.1587/elex.15.20180651).

[13] S. M. Ahsanuzzaman, et al.: "A low-volume hybrid step-down dc-dc converter based on the dual use of flying capacitor," IEEE Applied Power Electronics Conference and Exposition (2016) 2497 (DOI:
10.1109/APEC.2016.7468216).

[14] L. Chu, et al.: "10.5 A three-level single-inductor triple-output converter with an adjustable flying-capacitor technique for low output ripple and fast transient response," IEEE International SolidState Circuits Conference (2017) 186 (DOI: 10.1109/ISSCC.2017.7870323).

[15] S. Lee, et al.: "12.1 A $0.518 \mathrm{~mm} 2$ quasi-current-mode hysteretic buck DC-DC converter with $3 \mu$ s load transient response in $0.35 \mu \mathrm{m}$ BCDMOS," IEEE International Solid-State Circuits Conference (ISSCC) Digest of Technical Papers (2015) 1 (DOI: 10.1109/ISSCC.2015.7063002).

[16] W. Kim, et al.: "A Fully-Integrated 3-Level DC-DC Converter for Nanosecond-Scale DVFS," in IEEE Journal of Solid-State Circuits 47 (2012) 206 (DOI:10.1109/JSSC.2011.2169309).

[17] Y. Lei, et al.: "An Analytical Method to Evaluate and Design Hybrid Switched-Capacitor and Multilevel Converters," in IEEE Transactions on Power Electronics 33 (2018) 2227 (DOI: 10.1109/TPEL.2017.2690324).

[18] G. -S. Seo, et al.: "S-Hybrid Step-Down DC-DC ConverterAnalysis of Operation and Design Considerations," in IEEE Transactions on Industrial Electronics 67 (2020) 265 (DOI: 10.1109/TIE.2019.2897537).

[19] P. Hazucha, et al.: "A 233-MHz 80\%-87\% efficient four-phase DCDC converter utilizing air-core inductors on package," in IEEE Journal of Solid-State Circuits 40 (2005) 838 (DOI: 10.1109/JSSC.2004.842837).

[20] Y. Huh, et al.: "A Hybrid Structure Dual-Path Step-Down Converter With $96.2 \%$ Peak Efficiency Using 250-m $\Omega$ Large-DCR Inductor," in IEEE Journal of Solid-State Circuits 54 (2019) 959 (DOI: 10.1109/JSSC.2018.2882526).

[21] S. Zhen, et al.: "Design of Hybrid Dual-Path DC-DC Converter with Wide Input Voltage Efficiency Improvement," 2021 IEEE International Symposium on Circuits and Systems (2021) 1 (DOI: 10.1109/ISCAS51556.2021.9401442).

[22] K. Hata, et al.: "48V-to-12V Dual-Path Hybrid DC-DC Converter," IEEE Applied Power Electronics Conference and Exposition (2020) 2279 (DOI: 10.1109/APEC39645.2020.9124077).

[23] S. Shin, et al.: "High-Efficiency Hybrid Dual-Path Step-Up DC-DC Converter With Continuous Output-Current Delivery for Low Output Voltage Ripple," in IEEE Transactions on Power Electronics 35 (2020) 6025 (DOI: 10.1109/TPEL.2019.2954109).

[24] K. Hata, et al.: "Always-Dual-Path Hybrid DC-DC Converter Achieving High Efficiency at Around 2:1 Step-Down Ratio," IEEE Applied Power Electronics Conference and Exposition (2021) 1302 (DOI: 10.1109/APEC42165.2021.9487174).

[25] R. C. N. Pilawa-Podgurski, et al.: "Merged Two-Stage Power Converter With Soft Charging Switched-Capacitor Stage in $180 \mathrm{~nm}$ CMOS," in IEEE Journal of Solid-State Circuits 47 (2012) 1557 (DOI: 10.1109/JSSC.2012.2191325).

[26] Yuancheng Ren, et al.: "Analytical loss model of power MOSFET," in IEEE Transactions on Power Electronics 21 (2006) 310 (DOI:10.1109/TPEL.2005.869743).

[27] M. D. Seeman, et al.: "Analysis and Optimization of SwitchedCapacitor DC-DC Converters," in IEEE Transactions on Power Electronics 23 (2008) 841 (DOI: 10.1109/TPEL.2007.915182).

[28] M. Khorasani, et al.: "Low-power static and dynamic high-voltage CMOS level-shifter circuits," IEEE International Symposium on Circuits and Systems (2008) 1946 (DOI: 10.1109/ISCAS.2008.4541825).

[29] D. Liu, et al.: "Design of 370-ps Delay Floating-Voltage Level Shifters With 30-V/ns Power Supply Slew Tolerance," in IEEE Transactions on Circuits and Systems II: Express Briefs 63 (2016) 688 (DOI: 10.1109/TCSII.2016.2530902).

[30] Y. Moghe, et al.: "Nanosecond Delay Floating High Voltage Level Shifters in a 0.35 um HV-CMOS Technology," in IEEE Journal of $\begin{array}{lllll}\text { Solid-State Circuits } \quad 46 & \text { (2011) } 485 & \end{array}$ 10.1109/JSSC.2010.2091322). 